\title{
Eculizumab as rescue therapy in a renal transplant recipient with atypical hemolytic uremic syndrome: a case report
}

\author{
Joohyun Lee ${ }^{1}$, Jun Gyo Gwon ${ }^{1}$, Myung Gyu Kim², Cheol Woong Jung ${ }^{1}$ \\ ${ }^{1}$ Department of Surgery, Korea University College of Medicine, Seoul, Korea \\ ${ }^{2}$ Department of Internal Medicine, Korea University College of Medicine, Seoul, Korea
}

Background: Atypical hemolytic uremic syndrome (aHUS) after kidney transplantation (KT) can cause irreversible graft failure by over-activation of the alternative complement pathway. Eculizumab is a humanized monoclonal antibody against the human complement component $\mathrm{C} 5$ and has been used to treat aHUS. Herein, we report a case of a KT recipient with aHUS, which was salvaged with eculizumab.

Case report: A 61-year-old female with end-stage renal disease received a KT from a deceased donor. The surgery was performed successfully and serum creatinine $(\mathrm{sCr})$ level decreased to $0.74 \mathrm{mg} / \mathrm{dL}$ without acute rejection episodes. The patient received tacrolimus, mycophenolate mofetil and methylprednisolone as maintenance immunosuppressants. Four months after the $\mathrm{KT}$, the patient complained of general weakness and diarrhea. Her sCr level increased to $1.50 \mathrm{mg} / \mathrm{dL}$. The hemoglobin and platelet count decreased to $7.0 \mathrm{~g} / \mathrm{dL}$ and 20,000/ $\mu \mathrm{L}$, respectively. In addition, $\mathrm{LDH}$ increased to 3,207 IU/L. The kidney biopsy showed severe glomerular thrombotic microangiopathy. Under a diagnosis of aHUS, intense plasmapheresis was initiated. $\mathrm{However,} \mathrm{sCr}$ level increased to $4.32 \mathrm{mg} / \mathrm{dL}$ and anuria did not resolve for more than 10 days. Eculizumab was initiated as per usual protocol (900 mg weekly for 4 weeks followed by 1,200 mg every other week) at admission day 11 . Anti-meningococcal vaccination was administered before the first dose of eculizumab. Four weeks after eculizumab treatment, platelet count normalized to 236,000/ $\mu \mathrm{L}$ and $\mathrm{sCr}$ level declined to $2.05 \mathrm{mg} / \mathrm{dL}$. There were no significant side effects except for urinary tract infection controlled with antibiotics. Eculizumab was discontinued 3 months after treatment. Currently, 12 months after the presentation, renal allograft function has remained stable and there has been no evidence of aHUS recurrence.

Conclusions: Since aHUS after KT is associated with a high rate of allograft failure, prompt use of eculizumab along with plasmapheresis can be a valuable treatment to save renal allograft. 